\title{
Laser induced ablation of aluminum nanoparticle: a molecular dynamics study
}

\author{
Riser Fahdiran ${ }^{1,2, *}$, Erfan Handoko ${ }^{1}$, Iwan Sugihartono ${ }^{1}$, and Herbert M. Urbassek ${ }^{2, \#}$ \\ ${ }^{1}$ State University of Jakarta, Physics Department, Jalan Rawamangun Muka 13220 Jakarta Timur, Indonesia \\ ${ }^{2}$ University of Kaiserslautern, Physics Department and Research Center OPTIMAS, Erwin-Schrödinger-Straße 67663 Kaiserslautern, \\ Germany
}

\begin{abstract}
We investigate ablation of Aluminum nanoparticle due to ultrashort high-intensity laser pulse. Molecular dynamics simulation is used to follow the evolution of the system and observe thermodynamics together with cluster properties. The energy given from the laser to the system is $1.5 \mathrm{eV} /$ atom which is above ablation threshold and modelled using instantaneous and homogenous energization at the beginning of simulation. The evolution of the system shows a highly non-equilibrium state after the irradiation. Expansion of the sphere indicated that the system cannot survive from the temporary high tensile pressure that leads to ablation. While the high temperature consecutively responsible for the formation of cluster, it also indicated that the system is relaxed at the end of the simulation.
\end{abstract}

\section{Introduction}

The development of ultrashort high-intensities laser such as performed in FLASH or LCLS could bring a material into new regime thermodynamics state. It happens in an ultrafast time scale which leads to destruction of the materials [1]. The mechanism of interaction between a high-intensity laser with materials is still an open question. Since the system will be brought up to nonequilibrium state, the materials itself will not survive for energy above ablation threshold [2-7].

When a material irradiated by ultrashort highintensities laser, the evolution of the system will be, in general, depend on the energy that are deposited onto it surface [3]. For small energization, the material will melt and if it was given higher energy that constitutes a tensile pressure, then voids start to form. If the energization is even more energetic to produce stronger tensile pressure then voids will grow, material could be spallated and clusters will be formed. Molecular dynamics simulation gives the opportunity to follow these processes since the trajectory of the system is completely described by means of atomistic modelling.

Molecular dynamics simulation has been done for the case of thin film [2-6], Lennard-Jones nanoparticles [8] also the comparison between these two forms [9]. While for the case of smooth energization, such as two-temperature model, the energy transferred from the electronic to the atomic system have been done in bulk $[10,11]$ and thin film $[5,12]$. In this article, we explore the effect of instantaneous and homogenous energization applied to Aluminum nanoparticle.

\section{Simulation Method}

To study the mechanism of the materials due to ultrashort high-intensities laser irradiation, we use molecular dynamics (MD) simulation. Large-scale atomic/molecular massively parallel simulator was employed to carried out the simulation [13]. The material chosen for this simulation is Aluminum with interatomic potential are described by Liu, Ercolessi and Adams with cut-off radius $6.06 \AA$ [14].

The Aluminum nanoparticle has a diameter of 9.5 $\mathrm{nm}$ which consists of 28894 atoms. The initial temperature and pressure before the energization simulation are $0 \mathrm{~K}$ and $0 \mathrm{GPa}$ which has been relaxed for 20 ps. We employed instantaneous and homogenous energization to the system and follow the simulation up to $30 \mathrm{ps}$.

The laser considered to heated up the system in an ultrashort duration. This can be done for the case of $\mathrm{XUV} / \mathrm{X}$-ray laser where the energy transfer from the laser is in order of fs to ps. Within this simulation we considered the nanoparticle is energized at the beginning of the simulation with energy absorbed $\left(\mathrm{E}_{0}\right)$ by the system is $1.5 \mathrm{eV} /$ atom, which correspond to $\in=\mathrm{E}_{0} / \mathrm{E}_{\mathrm{coh}}$ $=(1.5 \mathrm{eV} /$ atom $) /(3.39 \mathrm{eV} /$ atom $)=0.44$, where it is already above ablation threshold [6].

We present the atomistic point of view of the simulation where we could observe all the mechanism mentioned before using OVITO [15]. Temperature and pressure as a function of time are also given to understand the evolution from thermodynamics point of view including virial term [16]. As a complementary we also provide the cumulative cluster distribution at the

\footnotetext{
* Corresponding author: riser-fahdiran@unj.ac.id

\# Corresponding author: urbassek@rhrk.uni-kl.de
} 
end of simulation using algorithm by Stoddard [17] and presented as in Upadhyay [18].

\section{Results and Discussion}

The evolution of the irradiated Aluminum nanoparticle are presented in Figure 1. At the beginning of the simulation, the nanoparticle is already heated up with initial temperature of $11500 \mathrm{~K}$ correspond to 1.5 eV/atom. After 1 ps, the system melts and some small clusters are escaped. At 4 ps, void started to form and grows larger until 8 ps. After all the sequences, system breaks up (spallated) at 20 ps. By the end of the simulation, $t=30 \mathrm{ps}$, clusters are formed with different sizes. This atomistic point of view is comparable to what have been done for the general case in Lennard-Jones material [8].

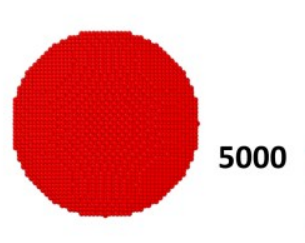

(a)

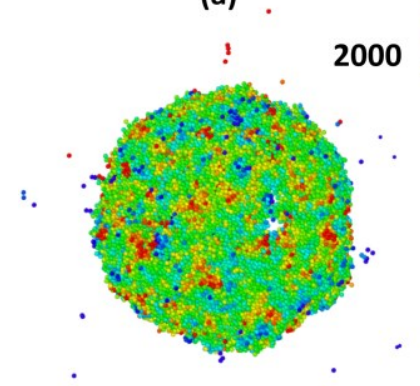

(c)

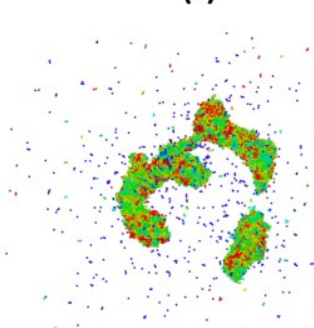

(e)

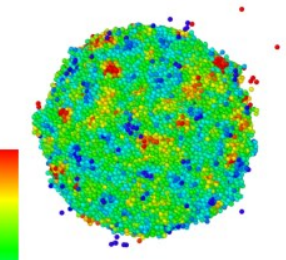

(b)

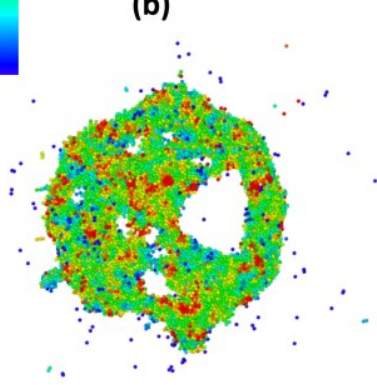

(d)

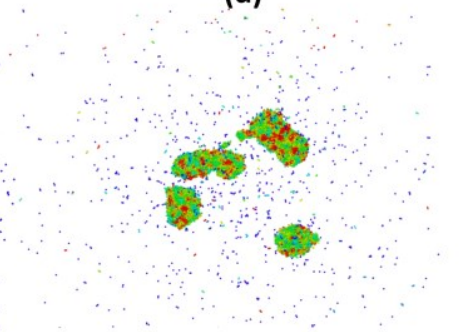

(f)
Fig. 1. System evolution with energization $1.5 \mathrm{eV} /$ atom. Atoms are colored based on local temperature in $\mathrm{K}$ (temperature color scale are given). Size are not to scale. (a) Aluminum nanoparticle at $\mathrm{t}=0 \mathrm{ps}$, initial temperature $11500 \mathrm{~K}$. (b) $\mathrm{t}=1$ ps, melting. (c) $t=4 p s$, void started to form. (d) $t=8$, void grows. (e) $t=20 \mathrm{ps}$, spallation. (f) $\mathrm{t}=30 \mathrm{ps}$, cluster.

Figure 2 shows the evolution of temperature with inset figure are for profile up to the first $1.5 \mathrm{ps}$. As can be seen from the figure, after the energization, the nanoparticle temperature is homogenized throughout the system. The initial temperature of $11500 \mathrm{~K}$ are already far away beyond critical temperature of Aluminum, $\mathrm{T}_{\mathrm{c}}=$ $5410 \mathrm{~K}[19]$. Within this regime of temperature, the nanoparticle is brought up into non-equilibrium state.
The first $1.5 \mathrm{ps}$ of the evolution, indicated that the system suffered for extreme temperature and pressure. After $1 \mathrm{ps}$, it melted completely, and some small clusters are moving away from the main body as in Figure 1(b). At this condition the temperature range are highly above the melting temperature of Aluminum, $\mathrm{T}_{\mathrm{m}}=933 \mathrm{~K}$. In this period also, the temperature is slightly oscillating above critical point where the system are boiled in an ultrashort time (up to $0.6 \mathrm{ps}$ ), $\mathrm{T}_{\mathrm{b}}=2623 \mathrm{~K}[20]$. Approaching $\mathrm{t}=1 \mathrm{ps}$, the system is heading to relax condition and maintain its temperature below critical point but above boiling temperature.

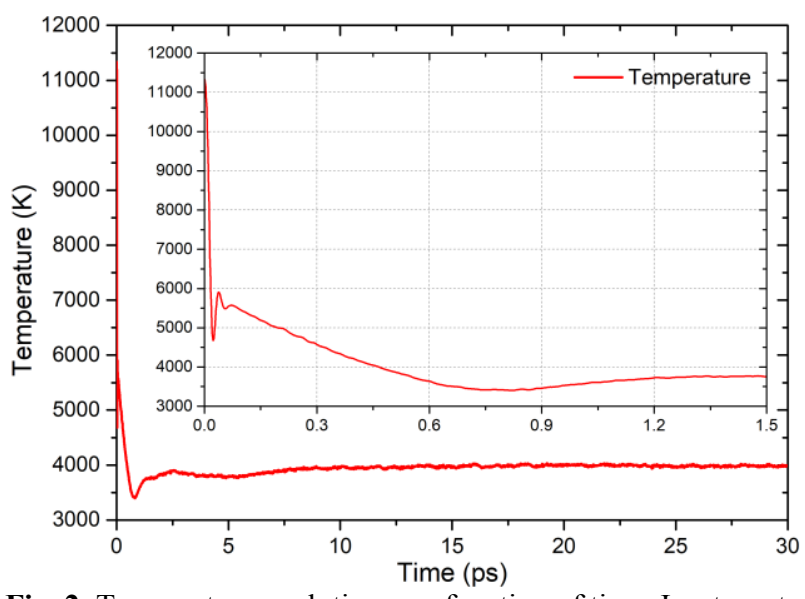

Fig. 2. Temperature evolution as a function of time. Inset: up to 1.5 ps profile.

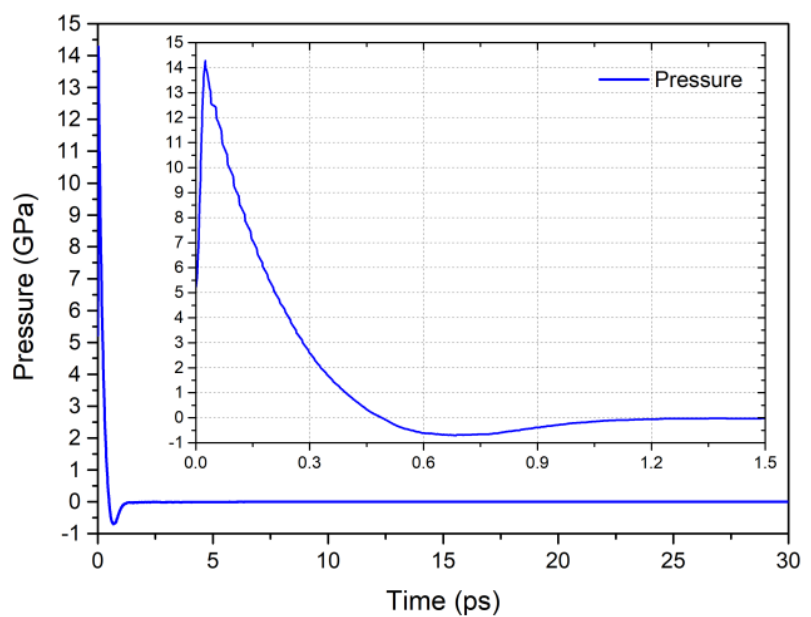

Fig. 3. Pressure evolution as a function of time. Inset: up to 1.5 ps profile.

As presented in Figure 3, at the very beginning, the nanoparticle system is suffered by compressive pressure which increasing up to more than $14 \mathrm{GPa}$. Hence, the nanoparticle is compressed, and the rarefaction wave started to travel into the system. This pressure then reaches the inner-part of nanoparticle and produce backward pressure. While this rarefaction also contributes in melting the nanoparticle, it also interacts with the upcoming rarefaction wave and create tensile pressure inside the system.

It can be seen from the pressure profile, at $\mathrm{t}=0.5 \mathrm{ps}$, pressure passes the value below $0 \mathrm{GPa}$ which indicated tensile pressure. This pressure is responsible for the system to spallated. As it was mentioned by Upadhyay 
and Urbassek [3], if the tensile pressure are strong enough then void will be formed. At $\mathrm{t}=4 \mathrm{ps}$, void is formed inside the nanoparticle and continue to grow. This indicated that the tensile pressure is strong enough, so the material cannot handle it. Evolving until $8 \mathrm{ps}$ it can be seen from Figure 1 (d) that multiple voids are created. As it was the case of thin films [3], when several voids are formed then the system will be spallated.

Continuing to follow the evolution of the system up to $20 \mathrm{ps}$, it can be recognized that the nanoparticle is spallated. The temperature at this time is around $3800 \mathrm{~K}$ which indicated that the system is boiled although it was in relax state. The pressure also relaxes at around $0 \mathrm{GPa}$, but since the nanoparticle is stable at this pressure, it continues to expand.

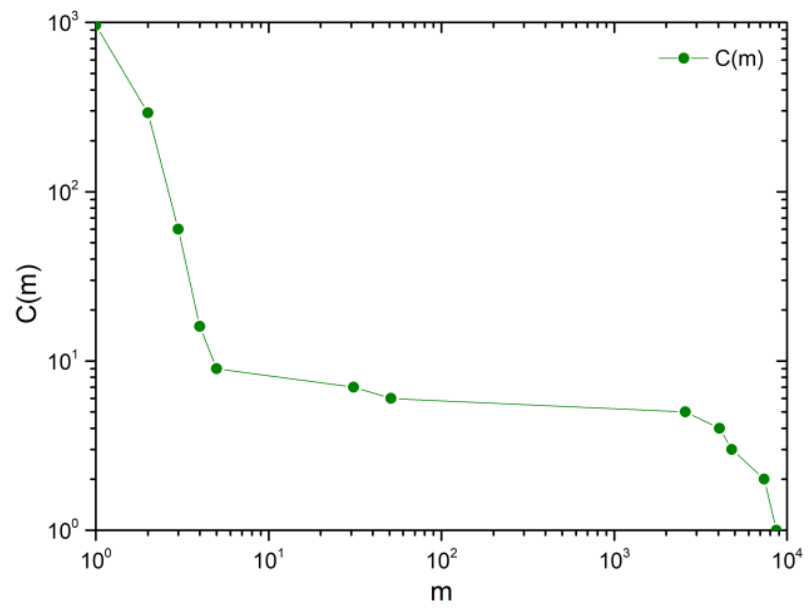

Fig. 4. Cumulative cluster distribution at the end of simulation, $\mathrm{t}=30$ ps.

At the end of simulation, $\mathrm{t}=30 \mathrm{ps}$, the system at the final state already fragmented into five big clusters (number of clusters $(\mathrm{m})>1000$ atoms) and several smaller clusters $(\mathrm{m}<100$ atoms). The main big clusters contribute to $70 \%$ of the nanoparticles, while the rest of smaller clusters is occupied the system especially the monomers $(m=1)$. The energy that is deposited into the system in the beginning of the simulation is responsible in forming this clusters. Within this simulation, where energy absorbed by the system is $1.5 \mathrm{eV} /$ atom, the nanoparticle is fragmented into several big clusters, while for even higher energization the system tend to boil up and bigger cluster cannot survived [8].

In summary, we studied the laser ablation of Aluminum nanoparticle by means of molecular dynamics simulation. The thermodynamics analysis which includes temperature and pressure evolution indicated that the system enters the regime of nonequilibrium state. As the energy absorbed by the laser is above the ablation threshold then the nanoparticle is spallated. Following the evolution of the system until the end of the state, it was indicated that the nanoparticle is fragmented resulting in several big clusters as well as small clusters around.

We acknowledged financial support by the Hibah Penelitian Kompetitif Unggulan Perguruan Tinggi (PKUPT) Universitas Negeri Jakarta.

\section{References}

1. M. Nicoul, F. Quirin, a. M. Lindenberg, a. Barty, D. M. Fritz, D. Zhu, H. Lemke, M. Chollet, D. a. Reis, J. Chen, S. Ghimire, M. Trigo, M. Fuchs, K. J. Gaffney, J. Larsson, T. Becker, S. Meyer, T. Payer, F. Meyer zu Heringdorf, M. Horn von Hoegen, M. Jerman, and K. Sokolowski-Tinten, EPJ Web Conf. 41, 04013 (2013).

2. A. K. Upadhyay and H. M. Urbassek, J. Phys. D. Appl. Phys. 38, 2933 (2005).

3. A. K. Upadhyay and H. M. Urbassek, J. Phys. Conf. Ser. 59, 68 (2007).

4. C. Wu and L. V. Zhigilei, Appl. Phys. A Mater. Sci. Process. 114, 11 (2014).

5. D. Ivanov and L. Zhigilei, Phys. Rev. B 68, 1 (2003).

6. A. K. Upadhyay, N. A. Inogamov, B. Rethfeld, and H. M. Urbassek, Phys. Rev. B 78, 1 (2008).

7. B. Rethfeld, D. S. Ivanov, M. E. Garcia, and S. I. Anisimov, J. Phys. D. Appl. Phys. 50, (2017).

8. R. Fahdiran and H. M. Urbassek, Eur. Phys. J. D 69, 35 (2015).

9. R. Fahdiran and H. M. Urbassek, Adv. Mater. Res. 1112, 120 (2015).

10. L. V. Zhigilei, Z. Lin, and D. S. Ivanov, J. Phys. Chem. C 113, 11892 (2009).

11. E. G. Gamaly, B. Luther-Davies, V. Z. Kolev, N. R. Madsen, M. Duering, and A. V. Rode, Laser Part. Beams 23, 167 (2005).

12. Y. Rosandi and H. M. Urbassek, Appl. Phys. A Mater. Sci. Process. 110, 649 (2013).

13. S. Plimpton, J. Comput. Phys. 117, 1 (1995).

14. X. Y. Liu, F. Ercolessi, and J. B. Adams, Model. Simul. Mater. Sci. Eng. 12, 665 (2004).

15. A. Stukowski, Model. Simul. Mater. Sci. Eng. 18, 015012 (2010).

16. T. J. Colla and H. M. Urbassek, Radiat. Eff. Defects Solids 142, 439 (1997).

17. S. D. Stoddard, J. Comput. Phys. 27, 291 (1978).

18. A. K. Upadhyay and H. M. Urbassek, Phys. Rev. B 73, 1 (2006).

19. V. V Zhakhovskii, K. Nishihara, S. I. Anisimov, and N. A. Inogamov, JETP Lett. 71, 167 (2000).

20. N. A. Inogamov, Y. V Petrov, S. I. Anisimov, A. M. Oparin, N. V Shaposhnikov, D. von der Linde, and J. Meyer-ter-Vehn, J. Exp. Theor. Phys. Lett. 69, 310 (1999). 